\title{
Heavy Nuclei and Super-heavy Elements
}

\section{S. G. Nilsson and S. E. Larsson, Lund \\ (Lund Institute of Technology)}

Nuclear fission was observed and correctly interpreted in the late autumn of 1938 and a theory of the fission process based on the liquid-drop model was put forth by N. Bohr and J.A. Wheeler in 1939. The theory considered the change in nuclear surface and Coulomb energies with shape. It predicted a one-hump smooth fission barrier. As it turned out, it correctly reproduced the magnitude of the fission barrier heights of about $10 \mathrm{MeV}$ or less in the actinide region of nuclei, although the detailed dependence on mass in the region was grossly in error.

\section{Fission Isomers}

An entirely new feature of fission, the occurrence of nuclei, which in an excited state are fissionable - the fission isomers, was discovered in 1962 by Polikanov and coworkers') in the Dubna laboratories in the Soviet Union. Presently more than 30 nucleides are known to have one or more fission isomeric states with half-lives in the range from $10^{-2}$ to $10^{-11} \mathrm{~s}$. The half-lives of these fissioning isomers are thus shorter by factors of $10^{20}$ to $10^{30}$ than the fission half-lives of the ground states of the same nucleides.

It was early suggested that the assumption of a coexistent shape of these nuclei might explain the existence of the fission isomeric states. This clearly implied a more structured potential-energy surface than the one consistent with the unmodified liquid-drop model. Subsequent analysis of the cross sections of neutron-induced fission has given strong support to this structured picture of the fission barrier (see Fig. 1). To provide an understanding of the occurrence of the second minimum the liquid-drop picture alone no more suffices. Instead one has to refer to shell structure effects.

\section{Shell Energy}

It is well known that a few nuclei such as ${ }_{8}^{16} \mathrm{O},{ }_{20}^{40} \mathrm{Ca},{ }_{20}^{48} \mathrm{Ca},{ }_{82}^{208} \mathrm{~Pb}$ are associated with particular stability. Since the days of the discovery of the shell model by M. Goeppert-Mayer, J.H. Jensen, P. Haxel and H.E. Suess, one says that these nuclei represent closed neutron and proton shells and thus correspond to the noble gases in the periodic table of atoms. The gain in binding energy relative to the gross trend in binding is of the order of $10 \mathrm{MeV}$ in these cases. The "average" with which one compares may then be represented more precisely by the liquid-drop model or the semiempirical mass formula, whose parameters are determined from a fit to all the available nuclear masses.

All the nuclei and magic numbers mentioned, reflect strong shell structure effects associated with spherical shapes. As seen from Fig. 2, showing the single-particle levels of a spheroidal oscillator potential, with the ec-

Fig. 1. The fission barrier of ${ }^{240} \mathrm{Pu}$ according to recent microscopic-macroscopic calculations. The abscissa is essentially labeled by the set of shapes indicated above the axis. Note the second minimum and the two-peak character of the barrier. Note that most of the energy liberated in the fission process is generated beyond the point of scission (separation point of fragments) by the Coulomb repulsion between the charged fragments.

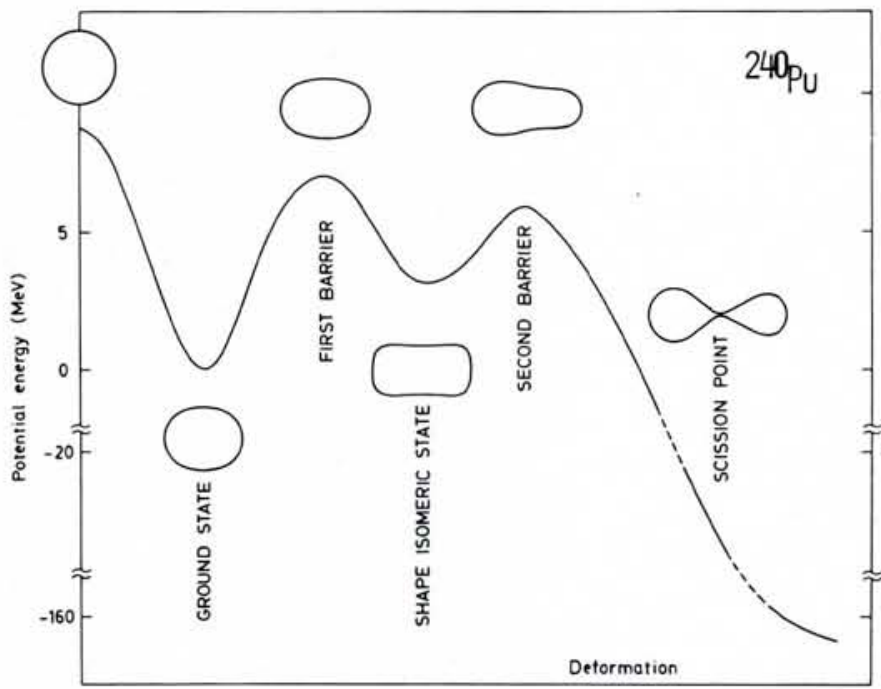

centricity parameter plotted on the abscissa, it is apparent that there is a strong tendency to shell formation not only for spherical shape $(\varepsilon=0)$ but also for spheroidal shapes with axis ratios of simple rational numbers as $1: 2$ and $1: 3$ etc.

This shell structure characteristic of non-spherical shapes has been recently emphasized by different authors $\left.{ }^{2-4}\right)$. It appears that the fission isomeric state is one of the first examples of a shape minimum related to the occurrence of a magic number associated with a 1:2 spheroidal shape.

A proof that the fission isomers indeed correspond to a state of radically different nuclear shape from that of the ground state was provided in the early seventies by a famous Munich experiment $\left.t^{5}\right)$. In this the isomeric rotational band of ${ }^{240} \mathrm{Pu}$ was populated and the internal conversion electrons of the transitions within the band $I=8 \rightarrow 6 \rightarrow 4 \rightarrow 2$ were observed (see Fig. 3). From these experiments the corresponding moment of inertia of the isomer band could be determined. It was seen to be more than double that of the ground-state rotational band. Detailed microscopic calculations of the moment of inertia came out consistent with the empirical inertia values on the basis of this $2: 1$ shape.

It is legitimate to ask for the magic numbers associated with this wildly distorted nuclear shape. The pure oscillator numbers are listed in Fig. 2. One number of particular interest would correspond to the neutron number $\mathrm{N}=140$ for the pure oscillator. $A$ more realistic potential than the pure oscillator which, for instance, includes a spin-orbit term, gives for neutrons, $N=146$ or 148 as a "deformed magic number", while for protons, there is a "deformed magic number" associated with $Z \simeq 92$. Indeed the isotopes of $\mathrm{U}, \mathrm{Np}, \mathrm{Pu}, \mathrm{Am}$ and $\mathrm{Cm}$, for which fission isomers are known, centre around these proton and neutron numbers.

\section{Macroscopic - Microscopic Method}

Until recently a large amount of theoretical work in nuclear spectroscopy of heavy nuclei was based on an extension of a deformed singleparticle potential constructed to fit, over large regions of nuclei, ground state properties as spins, moments, excitation spectra. This potential was 


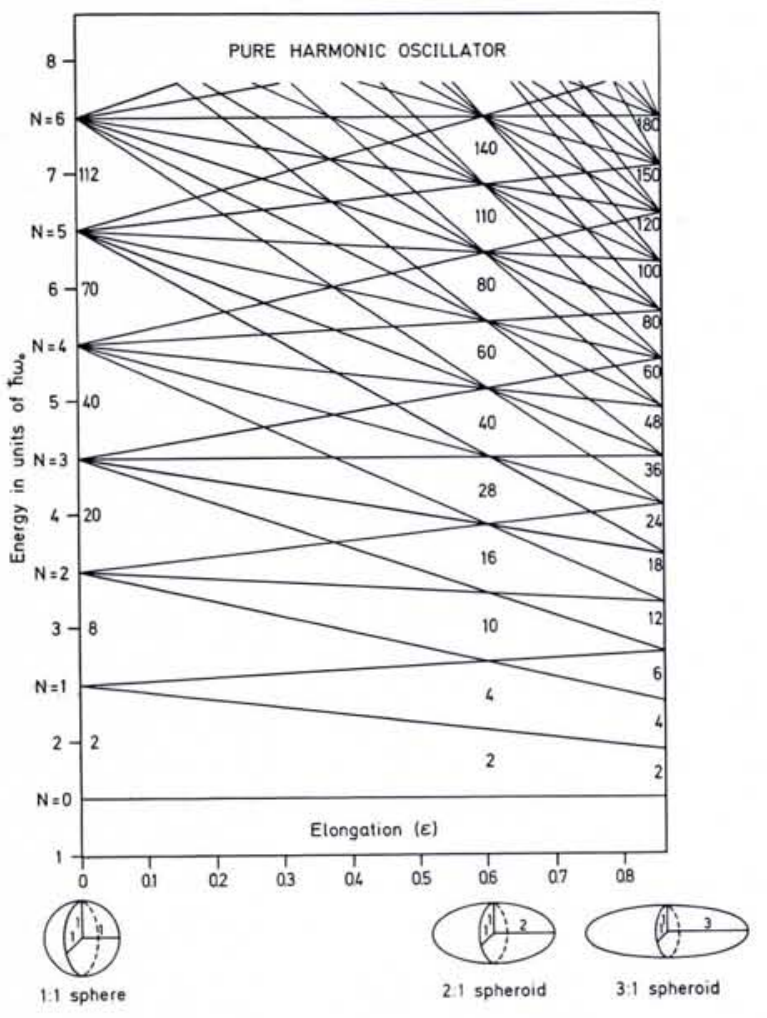

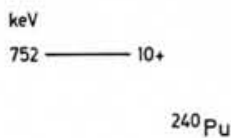

${ }^{240} \mathrm{Pu}$

$498-8+$

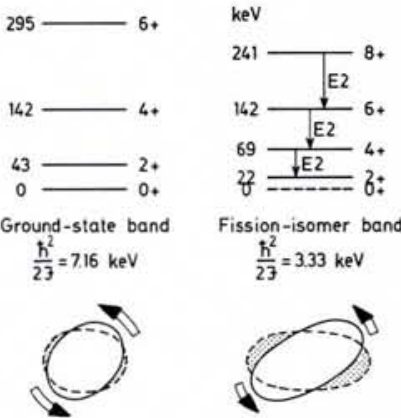

Fig. 2. (Left) Single-particle levels of pure spheroidal oscillator as function of eccentricity. The latter coordinate is visualised through the shapes below the abscissa. The formation of shells is apparent for axis ratios of $2: 1$ and $3: 1$ (and less clearly for $3: 2$ ).

Fig. 3. Two nuclear rotational-band spectra in units of keV for the two shapes of ${ }^{240} \mathrm{Pu}$. The left rotational band corresponds to the groundstate shape having a modest deformation, while the right band, corresponding to the shape isomeric state, has a large distortion corresponding to a $2: 1$ axis ratio. The latter band corresponds to more than twice the moment of inertia of that of the ground-state band and a correspondingly smaller level spacing. then generalized to describe more drastic shapes such as those presumably realized in the passage to fission. The method of calculating the total energy as a function of deformation simply as a sum of the occupied single-particle energies for each given shape was made reliable, however, first through the normalisation procedure suggested by Strutinsky ${ }^{3}$ ). Through the Strutinsky shell correction method it is assured, through the renormalisation, that "on the average" the summed single-particle energies behave with deformation as does the semi-empirical liquid-drop model.

In this way, the knowledge about the gross "macroscopic" behaviour of nuclear matter, contained in the liquid-drop model, is brought to a synthesis with the information contained in the single-particle shell model potentials. The method is therefore often described as the macroscopic-microscopic method. The results ${ }^{6}$ ) obtained have been remarkable. First, the reproduction of empirical ground state mass values is systematically improved relative to that of the semi-empirical mass formula. Quadrupole moments and hexadecapole moments in deformed regions are brought into good agreement with the extensive empirical data. One of the greatest achievements of the macroscopic-microscopic method has been the success already mentioned reached in the quantitative explanation of the fission shape isomer state. Another success has been the explanation of the fission fragment mass asymmetry as due to the instability of the barrier towards deformations involving mass asymmetric shapes. The second saddle point is found to be lowered in energy by roughly $2 \mathrm{MeV}$ by the inclusion of the octupole shape coordinate. In fact recent theoretical calculations claim to trace an "asymmetric valley" all the way to the point of scission.

\section{Hartree-Fock Method Applied to Heavy Nuclei}

The conceptual gap between the two-body force as derived from twonucleon scattering and the one-body potential used in the treatment of actual nuclei involving more than two or three nucleons has been narrowed - if not completely bridged - by the recent progress in Hartree-Fock calculations. A "phenomenological" twobody force, the so-called Skyrme force is found to reproduce nuclear masses, moments and spectra equally well as earlier the macroscopic-microscopic method turned out to do. When applied by a group in Orsay $^{7}$ ) to the more difficult problem of the description of the structured fission barrier, the Hartree-Fock calculations reproduce the inner of the two fission barrier peaks within 1 or $2 \mathrm{MeV}$. For the outer barrier, on the other hand, the solution has as yet not completely converged due to the insufficiency of the single-particle basis used.

We have thus a situation where most of the things that can be achieved by the macroscopic-microscopic method can also be achieved by a direct Hartree-Fock procedure. The drawback of the latter is, above all, the large increase in computer time needed. The conceptual success is also limited by the fact that the Skyrme type of interaction used is as yet "phenomenological" and not straight-forwardly derived from a "realistic" free nucleon-nucleon interaction. (The Hartree-Fock people denote the latter, as yet hypothetical, situation as "fully microscopic".)

\section{Superheavy Element Region}

The late 60's and early 70's saw several attempts in nuclear theory to make predictions concerning a nuclear island of stability associated with $\mathrm{Z}=114$ and $\mathrm{N}=184$ or thus about 40 nucleons in excess of the heaviest nuclei presently synthesized. The most recent predictions ${ }^{8}$ ) based on optimized single-particle potentials and a refitted liquid-drop model appear to give half-lives of at most 10$10^{5}$ years. These estimates lie already well below the limit of what is required for any nucleide surviving in terrestial matter to be observable. Thus already for this reason, this type of observation appears highly improbable. In addition, the calculation of fission barriers of elements on the probable r-process path to the superheavy island as shown in Fig. 4 indicates a trough of high instability isolating the island and extending also to $\mathrm{N}$-numbers near to $\mathrm{N}=184$. From these calculations it seems unlikely that, on the whole, superheavy ele- 
ments are generated by the r-process.

The recent Hartree-Fock calculations were extended in 1974 also to this superheavy region ${ }^{7}$ ). In part the results of the microscopic-macroscopic calculations are beautifully confirmed. Thus both $Z=114$ and $N=184$ are found to correspond to singleparticle gaps of a magnitude of about $2 \mathrm{MeV}$ (in near agreement with the predictions based on single-particle potentials or a little more than half the neutron gap $N=126$ and the proton gap $Z=82$ as found in ${ }^{208} \mathrm{~Pb}$ ). The longest half-life predicted is associated with the element $Z=110, N=184$, also in agreement with the early macroscopic-microscopic calculations. However, the half-life calculated on the basis of the Skyrme force is found to be of the order of days rather than tens of years or hundreds of thousands of years. The difference involved, reflects less a different singleparticle level structure around $\mathrm{Z}=$ 114 and $N=184$ and more the neutron-proton spatial correlation suggested by the Hartree-Fock solution.

\section{Synthesis of Superheavy Elements}

Whether indeed the superheavy island predicted, be associated with half-lives of the order of days or years, the half-lives involved should still allow ample time for a large number of physical and chemical studies, once these nucleides are synthesized. The half-lives indeed represent a remarkable increase in stability for some isotopes of elements $Z=110$ 114 relative to what appears indicated from the trend with $Z$ of the heavier elements so far synthesized. Thus, the half-life of the most long-lived even-even isotope of ${ }_{98} \mathrm{Cf}$ is about 1000 years, of ${ }_{100} \mathrm{Fm},{ }_{102} \mathrm{No}$ and ${ }_{104} \mathrm{Ku} / \mathrm{Rf}$ some days, some minutes and some seconds, respectively. A linear extrapolation of the logarithmic half-lives of fission and alpha-decay gives total half-lives for the even-even isotopes of the elements $Z=110,112$ and 114 of about $10^{-9}, 10^{-13}$ and $10^{-17} \mathrm{~s}^{7}$ ).

Early attempts to find superheavy elements in terrestial matter were made in many laboratories after 1968. Most of these were based on the study of possible spontaneous-fission events and aimed at the detection of energetic fission fragments or of a large number of neutrons emitted in coincidence with the fission fragments. Though spectacular and imaginative, these searches gave negative or inconclusive results. This holds true for the studies of terrestial matter as well as of matter from meteorites of cosmic cays. Most are now abandoned, but a few are still continued.

\section{Heavy Ion Physics}

Experimental efforts are now instead concentrated on the synthesis of superheavy elements through fusion or other types of heavy-ion reactions. Several heavy-ion accelerators are presently completed or near completion that allow an acceleration energy of $7 \mathrm{MeV}$ per nucleon or more of the heavy ions. This is roughly the energy needed to overcome the Coulomb barrier in the collision process involving heavy ions. One of the heaviest nucleides available in enough quantity for target material is ${ }_{96}^{248} \mathrm{Cm}$. In order to produce ${ }_{114}^{298}$ in a compound reaction there would be required an impossible ion projectile like ${ }_{18}^{50} \mathrm{Ar}$. The best available projectile in this situation is in fact ${ }_{20}^{48} \mathrm{Ca}$. The use of available projectiles and targets thus leads to products situated on the neutron deficient side of the superheavy island, where half-lives are much reduced. The fact that one always will end up on the neutron deficient side of the island reflects the bend of the stability line in the $(\mathrm{N}-\mathrm{Z})$ plane.

Still more adversary to the prospects of superheavy atom synthesis by heavy ion fusion is the fact that the compound nucleus forms at a high excitation energy and in the coolingdown process, the nucleus may emit a sequence of neutrons leading to an even more neutron deficient end nucleus, further away from the centre of the island of stability. More importantly, at the high excitation energy the fission barrier erodes as shell energy depletes with temperature. At this high excitation energy the ratio of fission to neutron widths weighs strongly in favour of fission.
Quite apart from the efforts to produce superheavy elements through the exploitation of new projectiles, targets and energies, the heavy-ion collision process is a subject of great interest in itself. Through these reactions also other new frontiers of nuclear physics are opened. One such new problem concerns the behaviour of heavy nuclei at high angular momentum or, in other words, what happens with the nucleus in terms of shape and internal structure as larger and larger units of angular momentum are deposited.

Work is also in progress on the problems of quantum electrodynamics associated with the electron shells of the quasi-atom formed transitionally in the collision process.

These interesting aspects fall, however, outside the scope set for this limited survey.

\section{References}

1. POLIKANOV, S.M., DRUIN, V.A., KARNAUKOV, V.A., MIKHEEV, V.L., PLEVE, A.A., SKOBOLEV, N.K., SUBBOTIN, V.G., TER-AKOPIAN, G.M. and FOMICHEV, V.A., Zh. Eksp. Teor. Fiz. (USSR) 42 (1962) 1464

2. GEILIKMAN, B.T., Proc. Int. Conf. Nucl. Structure, Kingston, 1960 (Univ. Press, Toronto), p. 874.

3. STRUTINSKY, V.M., Nucl. Phys. A95 (1967) 420.

4. SWIATECKI, W., private communication, 1970.

5. SPECHT, H.J., WEBER, J., KONECNY, E., and HEUNEMANN, D., Phys. Lett 41B (1972) 43

6. MOLLER, P. and NIX, J.R., Proc. Cont. Phys. and Chem. of Fission, Rochester 1973 (IAEA, Vienna, 1974), p. 103 and references therein.

7. BEINER, M., FLOCARD, H., VENERONI, M. and QUENTIN, P., Phys. Scripta 10A (1974) 84, and references therein.

8. RANDRUP, J., LARSSON, S.E., MOLLER, P., SOBICZEWSKI, A. and KUKASIAK, A., Phys. Scripta 10A (1974) 60

Fig. 4. Fission barrier heights calculated in the super-heavy element region of nuclei and in the region where the cosmological r-process of element synthesis is supposed to terminate. The names added to the map are introduced to facilitate the reference to specific areas. The elements so far produced artificially in Berkeley and in Dubna are found in the left lower corner, the predicted super-heavy island is found in the right upper corner. Element synthesis by means of the r-process may terminate south of the island in the region marked "Cape Hoyle".

\section{SYMMETRIC BARRIER HEIGHT (MeV)}

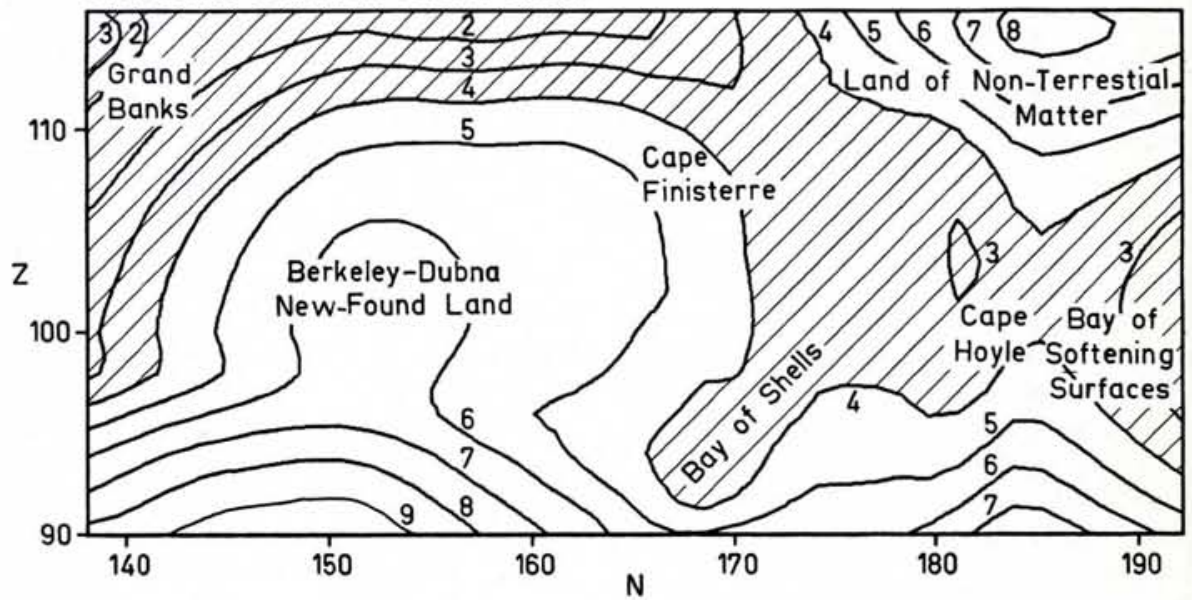

